\title{
On Green's function of Cauchy-Dirichlet problem for hyperbolic equation in a quarter plane
}

\author{
Makhmud Sadybekov and Bauyrzhan Derbissaly ${ }^{1,2^{*}}$
}

\section{"Correspondence:}

b.derbissaly@gmail.com

'Institute of Mathematics and

Mathematical Modeling, Pushkin

125, 050010, Almaty, Kazakhstan

${ }^{2}$ Al-Farabi Kazakh National

University, Al-Farabi 71, 050040,

Almaty, Kazakhstan

\begin{abstract}
The definition of a Green's function of a Cauchy-Dirichlet problem for the hyperbolic equation in a quarter plane is given. Its existence and uniqueness have been proven. Representation of the Green's function is given. It is shown that the Green's function can be represented by the Riemann-Green function.
\end{abstract}

Keywords: Hyperbolic equation; Initial-boundary value problem; Boundary condition; Green function

\section{Introduction}

This work is devoted to the substantiation of the Green's function method for solving the first initial-boundary value problem for a linear hyperbolic equation. The problem is considered in a quarter plane.

The Green's function method, well developed for elliptic and parabolic problems, is still little developed for hyperbolic problems. In our work, we introduce the definition of the Green's function for the hyperbolic problem (the first initial-boundary value problem), prove its existence and uniqueness, and also construct an integral representation of the solution to the problem using this Green's function.

The hyperbolic Green's function differs significantly from the Green's functions for elliptic or parabolic problems. In particular, the Green's function of a hyperbolic problem can have discontinuities along several characteristics of the equation. As we can see, in this regard, for each hyperbolic problem, the definition and substantiation of the Green's function must be carried out separately, and detailed studies in this direction are required.

If we consider the question of classical solvability of the following hyperbolic equation:

$$
u_{t t}-u_{x x}=f(x, t), \quad(x, t) \in S
$$

where $f \in C^{1}(\bar{S}), S=\{(x, t): 0<x<1, t>0\}$, with the initial

$$
u(x, 0)=u_{t}(x, 0)=0, \quad 0<x<1
$$

(c) The Author(s) 2021. This article is licensed under a Creative Commons Attribution 4.0 International License, which permits use, sharing, adaptation, distribution and reproduction in any medium or format, as long as you give appropriate credit to the original author(s) and the source, provide a link to the Creative Commons licence, and indicate if changes were made. The images or other third party material in this article are included in the article's Creative Commons licence, unless indicated otherwise in a credit line to the material. If material is not included in the article's Creative Commons licence and your intended use is not permitted by statutory regulation or exceeds the permitted use, you will need to obtain permission directly from the copyright holder. To view a copy of this licence, visit http://creativecommons.org/licenses/by/4.0/. 
Figure 1 The domain $D$

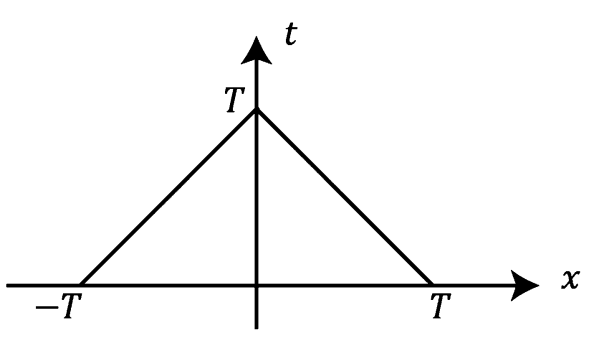

and boundary conditions

$$
u(0, t)=u(1, t)=0, \quad t>0,
$$

then by the Fourier method [1], we find a solution to problem (1.1)-(1.3) in the following form:

$$
u(x, t)=\int_{0}^{t} d \tau \int_{0}^{1} G(x, t ; \xi, \tau) f(\xi, \tau) d \xi
$$

where

$$
G(x, t ; \xi, \tau)=\sum_{k=1}^{\infty} \frac{2 \sin k \pi x}{k \pi} \sin k \pi(t-\tau) \sin k \pi \xi
$$

is the Green's function of problem (1.1)-(1.3). But from (1.5) we do not see what properties the Green's function of problem (1.1)-(1.3) has.

From the previous works related to the topic, the articles [2-25] should be noted.

First of all, we note the articles [2-5], in which, apparently, there were attempts to establish analogues of Sturm's comparison theorems for hyperbolic problems. However, it became clear that these extensions of classical results from the theory of ordinary differential operators to the hyperbolic problems require caution and special attention to boundary conditions and to the type of a domain in which the problem is being considered.

One of the explanations to this was given in the works of Kal'menov [6,7], in which eigenvalues and eigenfunctions of one class of the boundary value problems with shift for a wave equation in a characteristic triangle were calculated. It was shown that the eigenvalues of the problem have two series, real parts of which tend to $-\infty$ and to $+\infty$, respectively. Thus, even in the self-adjoint case, although the eigenvalues of the problem are real, the operator is not positive definite. This essentially distinguishes the hyperbolic problems from the boundary value problems for elliptic and parabolic equations. Note that these studies were continued in our subsequent works [8-10] as well.

To avoid the problems that have arisen, in [11] it was proposed to consider the construction of the Green's function for problems in the characteristic triangle $D=\{(x, t): t-T<$ $x<T-t, 0<t<T\}$ (see Fig. 1) for the wave equation

$$
u_{t t}-u_{x x}=f(x, t), \quad(x, t) \in D
$$


The problems on finding the coefficient $k$ with respect to the initial conditions

$$
\begin{aligned}
& u(x, 0)=k g(x), \quad u_{t}(x, 0)=0, \\
& \text { or } \quad-T<x<T, \\
& u(x, 0)=0, \quad u_{t}(x, 0)=k g(x),
\end{aligned}
$$

and the additional condition

$$
u(0, T)=0 \quad\left(\text { or } u_{t}(0, T)=0\right)
$$

were considered.

According to today's terminology, these problems are inverse problems of the recovery of coefficient.

When solving these problems, a construction resembling the Green's function arose. Using this function, in the domain $D$ we can solve problems (1.6)-(1.8) for the wave equation with the lower-order coefficient

$$
u_{t t}-u_{x x}+p(x, t) u=0, \quad(x, t) \in D .
$$

Obviously, having the Green's function, the solution to equation (1.9) with conditions (1.7), (1.8) can be reduced to the solution of an integral equation. Further, the results on comparing two positive solutions $u_{1} \leq u_{2}$ to problem (1.7), (1.8) for equation (1.9) with different potentials $p_{2} \leq p_{1}$ were obtained using the method from the monograph by Krasnoselskii [12].

The opportunity of using the Green's function to obtain such results prompted a more detailed research of the Green's function itself of hyperbolic problems in the characteristic triangle. Variants of various characteristic boundary value problems for hyperbolic equations and systems were considered in [13-22]. All cases of the considered problems were self-adjoint.

In the paper [23] one-dimensional wave equation with quadratic and hyperbolic nonlinearities was considered. Using the method of generalized separation of variables, it is shown that a hierarchy of nonlinear wave equations can be reduced to second-order nonlinear ordinary differential equations, to which Frasca's method is applicable. In [24] the Green's function was computed for the Dirichlet problem associated with the hyperbolic heat equation in the spatial interval $[0,1]$. It was shown that its Green's functions do not have to be continuous in all points of the domain. In [25] the authors considered the class of hypoelliptic operators

$$
L u \equiv \sum_{i, j=1}^{m_{0}} a_{i, j}(z) \partial_{x_{i}, x_{j}} u+\sum_{i, j=1}^{N} b_{i, j} x_{i} \partial_{x_{j}} u-\partial_{t} u,
$$

where $z=(x, t) \in \mathbb{R}^{N+1}, 0<m_{0} \leq N$, the coefficients $a_{i, j}$ belong to the space of vanishing mean oscillation functions, $B=\left(b_{i, j}\right)$ is a constant real matrix. In that paper they proved that a strong solution to the differential equation $L u=f$ with the known term $f$ in the Morrey space $L^{p, \lambda}$ belongs to a suitable Sobolev-Morrey space $S^{p, \lambda}$. 
Figure 2 The domain $\Gamma$

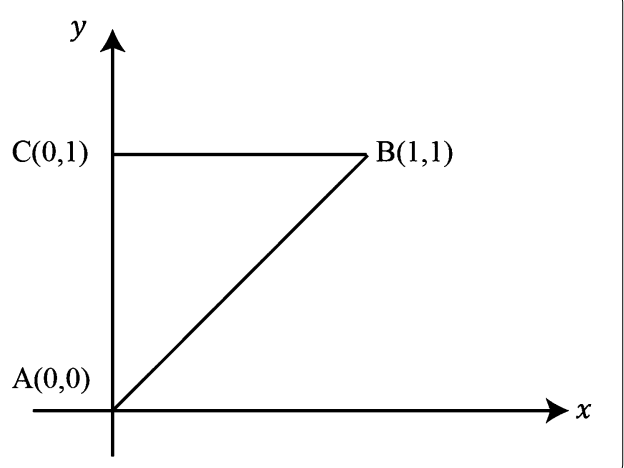

One of the most significant advances in constructing the Green's function of the characteristic boundary value problem was the work of Haws [20]. He considered the twodimensional hyperbolic equation

$$
u_{x y}+p(x, y) u=f(x, y), \quad(x, y) \in \Gamma
$$

in the characteristic triangle $\Gamma=\{(x, y): 0<x<y<1\}$ (see Fig. 2).

One of the following two conditions was chosen as a boundary condition on the noncharacteristic line $A B$ : either the boundary condition of the first kind

$$
u(x, x)=0, \quad 0 \leq x \leq 1,
$$

or the boundary condition of the second kind

$$
\left(u_{x}-u_{y}\right)(x, x)=0, \quad 0 \leq x \leq 1
$$

and the condition at the point $C$ was used:

$$
u(0,1)=0
$$

The problem was added by one more condition, which ensures the symmetry of the Green's function of the problem under consideration. As an example, it was proposed to use self-adjoint boundary conditions with shift from the works of Kal'menov [6, 7].

In the general case, the problem was only formulated, but not solved. For the special case $p(x, y) \equiv 0$, the definition of the Green's function was given and the method of its construction was indicated.

The solution to the problem was represented as the sum

$$
u(x, y)=\iint_{R_{i}} G^{i}(x, y ; \xi, \eta) f(\xi, \eta) d \xi d \eta
$$

where $G^{i}$ is the function defined in the subdomain $R_{i}$ (see Fig. 3).

Also, for the case of the symmetric coefficient

$$
p(x, y)=p(y, x),
$$


Figure 3 The subdomains $R_{i}$

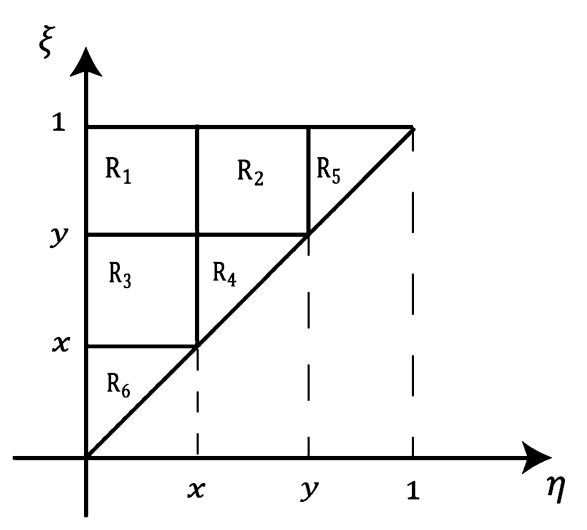

the possibility of constructing the Green's function was justified using the Riemann-Green function. However, the method used does not allow one to abandon the symmetry condition of potential (1.11).

The author also considered a hyperbolic equation with lower-order coefficients

$$
u_{x y}+a u_{x}+b u_{y}+c u=f(x, y), \quad(x, y) \in \Gamma .
$$

However, only the case of constant coefficients $a, b, c$ was considered.

The problem of constructing the Green's function of a characteristic boundary value problem with arbitrary coefficients (without using the symmetry condition (1.11)) was singled out as an unsolved problem that is interesting for further consideration.

This problem still remains unsolved.

\section{Formulation of the problem}

Let $Q=\{(x, t): x>0, t>0\}$. The following hyperbolic equation is considered in $Q$ :

$$
\begin{aligned}
L u \equiv & \frac{\partial^{2} u(x, t)}{\partial t^{2}}-\frac{\partial^{2} u(x, t)}{\partial x^{2}}+a_{1}(x, t) \cdot \frac{\partial u(x, t)}{\partial x} \\
& +b_{1}(x, t) \cdot \frac{\partial u(x, t)}{\partial x}+c_{1}(x, t) \cdot u(x, t)=F(x, t), \quad(x, t) \in Q,
\end{aligned}
$$

with the initial conditions

$$
u(x, 0)=T(x), \quad \frac{\partial u}{\partial t}(x, 0)=N(x), \quad x>0,
$$

and the boundary condition

$$
u(t, 0)=\Phi(t), \quad t>0 .
$$

It is well known that this problem is correct, both in the sense of classical and generalized solutions. We are interested in the question of integral form of the solution of problem (2.1)-(2.3). We show that the solution to the problem can be written in terms of the Green's function, the definition of which we introduce. 
In the characteristic coordinates $\xi=x+t, \eta=x-t$ equation (2.1) has the form

$$
\frac{\partial^{2} u}{\partial \xi \partial \eta}+a(\xi, \eta) \cdot \frac{\partial u}{\partial \xi}+b(\xi, \eta) \cdot \frac{\partial u}{\partial \eta}+c(\xi, \eta) \cdot u=f(\xi, \eta), \quad(\xi, \eta) \in \Omega
$$

and initial conditions (2.2) have the form

$$
u(\xi, \xi)=\tau(\xi), \quad\left(\frac{\partial u}{\partial \xi}-\frac{\partial u}{\partial \eta}\right)(\xi, \xi)=v(\xi), \quad \xi>0
$$

and boundary condition (2.3) will change to

$$
u(-\eta, \eta)=\varphi(\eta), \quad \eta \leq 0
$$

We will assume that $a, b \in C^{1}(\bar{\Omega}) ; c, f \in C(\bar{\Omega}) ; \varphi \in C^{1}((-\infty, 0]) ; v \in C([0,+\infty)) ; \tau \in$ $C^{1}([0,+\infty)) ; \varphi^{\prime}(0)=-v(0), \varphi(0)=\tau(0)$.

The aim is to construct a Green's function and a solution to problem (2.4)-(2.6).

\section{Proof of correctness of problem (2.4)-(2.6)}

For the sake of completeness, we present here a proof of the correctness of the considered problem (2.4)-(2.6).

Let us call a function from the class $u(\xi, \eta), u_{\xi \eta} \in C(\bar{\Omega})$ a regular solution to the problem, converting equation (2.4), initial conditions (2.5), and boundary condition (2.6) into an identity.

Theorem 3.1 Let $a, b \in C^{1}(\bar{\Omega}) ; c, f \in C(\bar{\Omega}) ; \varphi \in C^{1}((-\infty, 0]) ; \quad v \in C([0,+\infty)) ; \tau \in$ $C^{1}([0,+\infty)) ; \varphi^{\prime}(0)=-v(0), \varphi(0)=\tau(0)$. Then problem $(2.4)-(2.6)$ has a unique regular solution.

\subsection{Proof of existence of solution of problem (2.4)-(2.6)}

Let

$$
u(\xi, \eta)=\zeta(\xi, \eta) \cdot \omega(\xi, \eta) .
$$

Then (2.4) has the form

$$
\begin{aligned}
& \frac{\partial^{2} \zeta}{\partial \xi \partial \eta} \cdot \omega+\frac{\partial^{2} \omega}{\partial \xi \partial \eta} \cdot \zeta+\left[\frac{\partial \zeta}{\partial \xi}+b \zeta\right] \cdot \frac{\partial \omega}{\partial \eta}+\left[\frac{\partial \zeta}{\partial \eta}+a \zeta\right] \cdot \frac{\partial \omega}{\partial \xi} \\
& +\left[b \frac{\partial \zeta}{\partial \eta}+a \frac{\partial \zeta}{\partial \xi}+c \zeta\right] \cdot \omega=f .
\end{aligned}
$$

We choose $\zeta(\xi, \eta)$ in such a way that

$$
\frac{\partial \zeta(\xi, \eta)}{\partial \xi}+b(\xi, \eta) \cdot \zeta(\xi, \eta)=0
$$

holds. From (3.3) we get

$$
\zeta(\xi, \eta)=\exp \left(-\int_{0}^{\xi} b(s, \eta) d s\right)
$$


Figure 4 The domain $\Omega_{(\xi \eta)}$, when $\eta>0$

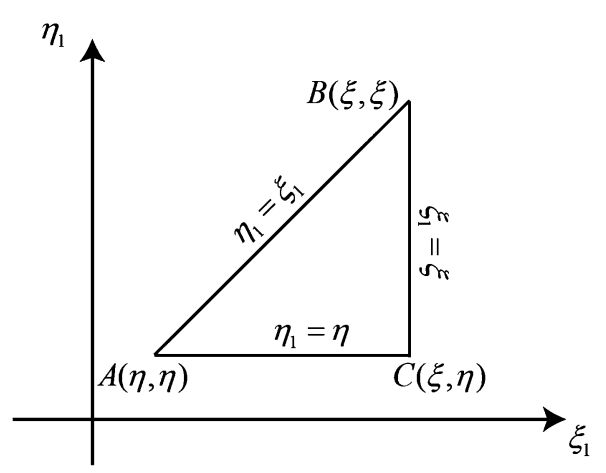

Dividing equation (3.2) by $\zeta$, we have the following problem:

$$
\begin{aligned}
& \frac{\partial^{2} \omega}{\partial \xi \partial \eta}+a_{2}(\xi, \eta) \cdot \frac{\partial \omega}{\partial \xi}+c_{2}(\xi, \eta) \cdot \omega=f_{2}, \quad(\xi, \eta) \in \Omega, \\
& \omega(\xi, \xi)=\tau_{2}(\xi), \quad\left(\frac{\partial \omega}{\partial \xi}-\frac{\partial \omega}{\partial \eta}\right)(\xi, \xi)=v_{2}(\xi), \quad \xi>0, \\
& \omega(-\eta, \eta)=\varphi_{2}(\eta), \quad \eta \leq 0,
\end{aligned}
$$

where

$$
\begin{aligned}
& a_{2}=\frac{1}{\zeta} \cdot \zeta_{\eta}+a, \\
& c_{2}=\frac{1}{\zeta}\left(\zeta_{\xi \eta}+a \zeta_{\xi}+b \zeta_{\eta}+c\right), \quad \tau_{2}(\xi)=\frac{\tau(\xi)}{\zeta(\xi, \xi)}, \quad \varphi_{2}(\eta)=\frac{\varphi(\eta)}{\zeta(-\eta, \eta)}, \\
& \nu_{2}(\xi)=\frac{v(\xi)}{\zeta(\xi, \xi)}-\frac{\tau_{2}(\xi)\left(\zeta_{\xi}(\xi, \xi)-\zeta_{\eta}(\xi, \xi)\right)}{\zeta(\xi, \xi)}, \quad f_{2}(\xi, \eta)=\frac{f(\xi, \eta)}{\zeta(\xi, \eta)}
\end{aligned}
$$

Let us introduce a new notation

$$
\frac{\partial \omega}{\partial \xi}=v
$$

Then equation (3.5) is equivalent to the following system of equations:

$$
\left\{\begin{array}{l}
\frac{\partial v}{\partial \eta}=f_{2}(\xi, \eta)-a_{2}(\xi, \eta) \cdot v(\xi, \eta)-c_{2}(\xi, \eta) \cdot \omega(\xi, \eta) \\
\frac{\partial \omega}{\partial \xi}=v(\xi, \eta)
\end{array}\right.
$$

In the domain $\Omega$, take an arbitrary point $C(\xi, \eta)$ and draw the characteristics $C B, C D, C A$ through it to the boundary of the domain $\Omega$ (see Figs. 4, 5). Integrating the first equation of system (3.8) by $D C, A C$, the second by $B C$, and using conditions (3.6), (3.7), we get

$$
\left\{\begin{array}{l}
v(\xi, \eta)=\psi(\xi)-\int_{\eta}^{\xi}\left[f_{2}\left(\xi, \eta_{1}\right)-a_{2}\left(\xi, \eta_{1}\right) v\left(\xi, \eta_{1}\right)-c_{2}\left(\xi, \eta_{1}\right) \omega\left(\xi, \eta_{1}\right)\right] d \eta_{1} \\
\omega(\xi, \eta)=\tau_{1}(\eta)+\int_{|\eta|}^{\xi} v(\xi, \eta) d \xi_{1}, \quad(\xi, \eta) \in \Omega,
\end{array}\right.
$$


Figure 5 The domain $\Omega_{(\xi \eta)}$, when $\eta<0$

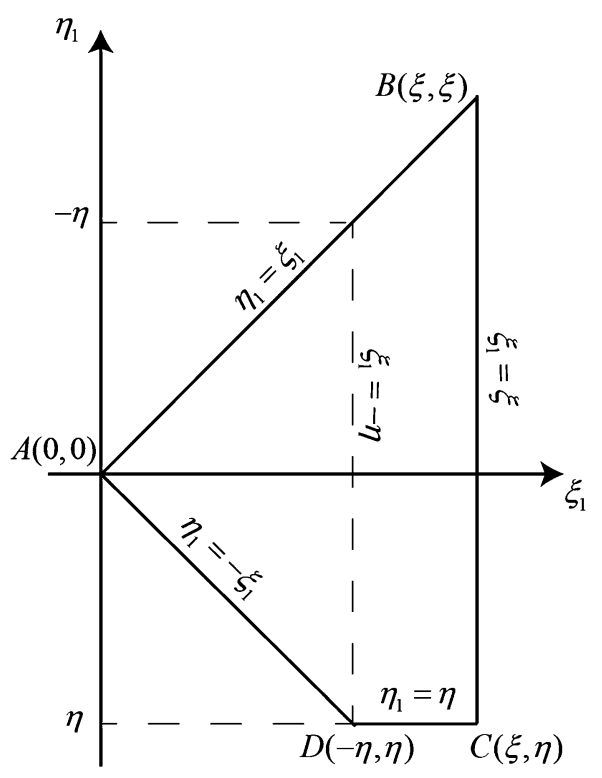

where

$$
\begin{aligned}
& \psi(\xi)=\frac{1}{2}\left(\tau_{2}^{\prime}(\xi)+v_{2}(\xi)\right), \\
& \tau_{1}(\eta)= \begin{cases}\tau_{2}(\eta) & \text { when } \eta>0 \\
\varphi_{2}(\eta) & \text { when } \eta \leq 0\end{cases}
\end{aligned}
$$

It is easy to show that, if $v(\xi, \eta), \omega(\xi, \eta)$ are solutions to system (3.9), then $\omega(\xi, \eta)$ is a solution to problem (3.5)-(3.7). Therefore, system (3.9) is equivalent to problem (3.5)(3.7).

We will search the solution to system (3.9) using the method of successive approximations. Let $M$ be an arbitrary positive number. $\Omega_{M}=\Omega \cap\{\xi<M\}$ and for points $(\xi, \eta) \in \Omega_{M}$ choose an initial approximation

$$
v_{0}(\xi, \eta)=\psi(\xi), \quad \omega_{0}(\xi, \eta)=\tau_{1}(\eta)
$$

We construct the following approximation using the formulas:

$$
\left\{\begin{aligned}
v_{n}(\xi, \eta)= & \psi(\xi)-\int_{\eta}^{\xi}\left[f_{2}\left(\xi, \eta_{1}\right)-a_{2}\left(\xi, \eta_{1}\right) v_{n-1}\left(\xi, \eta_{1}\right)\right. \\
& \left.-c_{2}\left(\xi, \eta_{1}\right) \omega_{n-1}\left(\xi, \eta_{1}\right)\right] d \eta_{1}, \\
\omega_{n}(\xi, \eta)= & \tau_{1}(\eta)+\int_{|\eta|}^{\xi} v_{n-1}\left(\xi_{1}, \eta\right) d \xi_{1}, \quad(\xi, \eta) \in \Omega_{M}
\end{aligned}\right.
$$

We prove the uniform convergence of sequences $v_{n}, \omega_{n}$ in a closed domain $\bar{\Omega}_{M}$. Let us make up the differences

$$
\left\{\begin{array}{l}
v_{n+1}-v_{n}=\int_{\eta}^{\xi}\left[a_{2}\left(v_{n}\left(\xi, \eta_{1}\right)-v_{n-1}\left(\xi, \eta_{1}\right)\right)+c_{2}\left(\omega_{n}\left(\xi, \eta_{1}\right)-\omega_{n-1}\left(\xi, \eta_{1}\right)\right)\right] d \eta_{1} \\
\omega_{n+1}-\omega_{n}=\int_{|\eta|}^{\xi}\left[v_{n}\left(\xi_{1}, \eta\right)-v_{n-1}\left(\xi_{1}, \eta\right)\right] d \xi_{1}, \quad(\xi, \eta) \in \Omega_{M} .
\end{array}\right.
$$


Show that differences $\left|v_{n}-v_{n-1}\right|$ and $\left|\omega_{n}-\omega_{n-1}\right|$ satisfy the inequalities

$$
\begin{aligned}
& \left|v_{n}-v_{n-1}\right| \leq K^{n} \cdot A \cdot \frac{(\xi-\eta)^{n}}{n !}, \\
& \left|\omega_{n}-\omega_{n-1}\right| \leq K^{n} \cdot A \cdot \frac{(\xi-\eta)^{n}}{n !}
\end{aligned}
$$

where

$$
\begin{aligned}
& K=\max _{\bar{\Omega}_{M}}\left[1,\left|a_{2}\right|+\left|c_{2}\right|\right], \\
& A=\left\|f_{2}\right\|_{C\left(\bar{\Omega}_{M}\right)}+\|\psi\|_{C([0, M])}+\left\|\tau_{1}\right\|_{C([-M, M])},
\end{aligned}
$$

where $\tau_{1}(\eta), \psi(\xi)$ are defined in (3.10), (3.11). We prove the validity of inequalities (3.14) by mathematical induction. For $n=1$, as is easy to see from (3.12), the estimates of (3.14) are correct.

We show that these inequalities will remain valid when $n$ is replaced with $n+1$. From equality (3.13), according to the classical method, we have

$$
\begin{aligned}
\left|v_{n+1}-v_{n}\right| & \leq \int_{\eta}^{\xi}\left(\left|a_{2}\right|+\left|c_{2}\right|\right) \cdot K^{n} \cdot A \cdot \frac{\left(\xi-\eta_{1}\right)^{n}}{n !} d \eta_{1} \\
& \leq K^{n+1} \cdot A \cdot \int_{\eta}^{\xi} \frac{\left(\xi-\eta_{1}\right)^{n}}{n !} d \eta_{1}=\frac{K^{n+1}}{(n+1) !} \cdot A \cdot(\xi-\eta)^{n+1} \\
\left|\omega_{n+1}-\omega_{n}\right| & \leq \int_{|\eta|}^{\xi} K^{n} \cdot A \cdot \frac{\left(\xi_{1}-\eta\right)^{n}}{n !} d \xi_{1} \leq K^{n+1} \cdot A \cdot \int_{|\eta|}^{\xi} \frac{\left(\xi_{1}-\eta\right)^{n}}{n !} d \xi_{1} \\
& =\frac{K^{n+1}}{(n+1) !} \cdot A \cdot(\xi-\eta)^{n+1}-\frac{K^{n+1}}{(n+1) !} \cdot A \cdot(|\eta|-\eta)^{n+1} \\
& \leq \frac{K^{n+1}}{(n+1) !} \cdot A \cdot(\xi-\eta)^{n+1}
\end{aligned}
$$

Estimates (3.14) show absolute and uniform convergence over $\bar{\Omega}_{M}$ of the following series:

$$
v_{0}+\sum_{n=1}^{\infty}\left(v_{n}-v_{n-1}\right), \omega_{0}+\sum_{n=1}^{\infty}\left(\omega_{n}-\omega_{n-1}\right),
$$

members of which are less than the absolute value of the members of the uniformly converging series

$$
A+A \cdot \sum_{n=1}^{\infty} K^{n} \frac{(\xi-\eta)^{n}}{n !}=A \cdot \exp (K(\xi-\eta))
$$

Consequently, successive approximations of $v_{n}, \omega_{n}$ on $\bar{\Omega}_{M}$ uniformly tend, respectively, to certain limits of $v, \omega$, which are continuous on $\bar{\Omega}_{M}$. Passing to the limit in equalities (3.12), we obtain that the limit functions of $v(\xi, \eta), \omega(\xi, \eta)$ satisfy system (3.9). In this case, the functions $v, \omega$ are continuous on $\bar{\Omega}_{M}$. Since we have proved the existence of the solution in $\Omega_{M}$ for any $M$, the solution exists in the entire $\Omega$ domain. The solution to problem $(2.4)-(2.6)$ is found by substituting $\omega, \varphi$ for (3.1). 


\subsection{Proof of uniqueness of the solution to problem (3.5)-(3.7)}

Assume that system (3.9) has different solutions $v_{1}, \omega_{1}, v_{2}, \omega_{2}$. Denote $V=v_{1}-v_{2}, W=$ $\omega_{1}-\omega_{2}$. Then $V, W$ satisfy the following system of equations:

$$
\left\{\begin{array}{l}
V(\xi, \eta)=\int_{\eta}^{\xi}\left[a_{2}\left(\xi, \eta_{1}\right) \cdot V\left(\xi, \eta_{1}\right)+c_{2}\left(\xi, \eta_{1}\right) \cdot W\left(\xi, \eta_{1}\right)\right] d \eta_{1} \\
W(\xi, \eta)=\int_{|\eta|}^{\xi} V\left(\xi_{1}, \eta\right) d \xi_{1}, \quad(\xi, \eta) \in \Omega_{M} .
\end{array}\right.
$$

Let us prove that $V=W \equiv 0$. Functions $V, W$ are continuous and bounded as the differences of continuous functions in a closed domain $\bar{\Omega}_{M}$. Therefore, there is a positive constant $B$ such that

$$
|V(\xi, \eta)| \leq B, \quad|W(\xi, \eta)| \leq B, \quad(\xi, \eta) \in \Omega_{M}
$$

Then by (3.16) we have

$$
\begin{aligned}
& |V| \leq B \int_{\eta}^{\xi}\left(\left|a_{2}\left(\xi, \eta_{1}\right)\right|+\left|c_{2}\left(\xi, \eta_{1}\right)\right|\right) d \eta_{1} \leq K \frac{(\xi-\eta)}{1 !}, \\
& |W| \leq B \int_{|\eta|}^{\xi} B d \xi_{1} \leq K \cdot \frac{(\xi-\eta)}{1 !}
\end{aligned}
$$

where $K$ is defined in (3.15). By mathematical induction, for any $n$, we obtain the following estimates:

$$
|V| \leq B K^{n} \frac{(\xi-\eta)^{n}}{n !}, \quad|W| \leq B K^{n} \frac{(\xi-\eta)^{n}}{n !} .
$$

Since these inequalities are met for any $n$, then it follows that $V=W \equiv 0$, i.e., $v_{1}=v_{2}, \omega_{1}=$ $\omega_{2}$.

\subsection{Proof of stability of the solution to problem (3.5)-(3.7)}

In order to prove the stability of the solution to problem (3.5)-(3.7), we need a stability estimate for $\omega$. Since

$$
\omega(\xi, \eta)=\lim _{N \rightarrow \infty} \omega_{N}=\lim _{N \rightarrow \infty}\left[\omega_{0}+\sum_{n=1}^{N}\left(\omega_{n}-\omega_{n-1}\right)\right]=\omega_{0}+\sum_{n=1}^{\infty}\left(\omega_{n}-\omega_{n-1}\right),
$$

then using the estimate of (3.14), from (3.17), we get

$$
|\omega(\xi, \eta)| \leq A \cdot \sum_{n=0}^{\infty} \frac{K^{n}}{n !}(\xi-\eta)^{n}=A \cdot \exp (2 K M)
$$

where $A$ is defined in (3.15). Using equality (3.15), from (3.18), we have

$$
|\omega(\xi, \eta)| \leq \exp (2 K M) \cdot\left(\left\|f_{1}\right\|_{C\left(\bar{\Omega}_{M}\right)}+\|\psi\|_{C([0, M])}+\left\|\tau_{1}\right\|_{C([-M, M])}\right) .
$$


Hence, using (3.10), (3.11), we obtain the estimate of the stability of the solution to problem (3.5)-(3.7):

$$
\begin{aligned}
\|\omega\|_{C\left(\Omega_{M}\right)} \leq & \exp (2 K M) \\
& \times\left(\left\|f_{1}\right\|_{C\left(\bar{\Omega}_{M}\right)}+\left\|v_{2}\right\|_{C([0, M])}+\left\|\tau_{2}\right\|_{C^{1}([0, M])}+\left\|\varphi_{2}\right\|_{C([-M, 0])}\right) .
\end{aligned}
$$

\section{On the Riemann function of equation (2.4)}

It is well known that the Riemann-Green function $R\left(\xi, \eta ; \xi_{1}, \eta_{1}\right)$ is not defined in the entire domain $\Omega \times \Omega$, but only for those points $\left(\xi_{1}, \eta_{1}\right) \in \Omega$, for which $|\eta|<\xi_{1},-\xi<\eta_{1}<\xi$. And for the remaining points of the domain $\Omega \times \Omega$, the Riemann-Green function is not uniquely determined. For our further constructions, it is important for us to use the RiemannGreen function defined at all points of the domain $\Omega \times \Omega$, for which $\eta_{1}<-\xi$.

For further reasoning, we need to fulfill some relations between the coefficients $a(\xi, \eta)$ and $b(\xi, \eta)$ on the border $\xi=-\eta$. For this purpose, in equation (2.4) let us replace the function

$$
u(\xi, \eta)=U(\xi, \eta) \cdot \gamma(\eta) \cdot \mu(\xi) .
$$

Then with respect to the new unknown function $U(\xi, \eta)$, we get the equation

$$
\frac{\partial^{2} U}{\partial \xi \partial \eta}+\widehat{a}(\xi, \eta) \frac{\partial U}{\partial \xi}+\widehat{b}(\xi, \eta) \frac{\partial U}{\partial \eta}+\widehat{c}(\xi, \eta) U=\widehat{f}, \quad(\xi, \eta) \in \Omega
$$

where

$$
\begin{aligned}
& \widehat{a}=\frac{1}{\gamma(\eta)} \cdot\left(\gamma^{\prime}(\eta)+a(\xi, \eta) \gamma(\eta)\right), \quad \widehat{b}=\frac{1}{\mu(\xi)} \cdot\left(\mu^{\prime}(\xi)+b(\xi, \eta) \mu(\xi)\right), \\
& \widehat{c}=\frac{\gamma^{\prime}(\eta) \mu^{\prime}(\xi)}{\gamma(\eta) \mu(\xi)}+a(\xi, \eta) \frac{\mu^{\prime}(\xi)}{\mu(\xi)}+b(\xi, \eta) \frac{\gamma^{\prime}(\eta)}{\gamma(\eta)}+c(\xi, \eta), \quad \widehat{f}=\frac{f}{\gamma(\eta) \mu(\xi)} .
\end{aligned}
$$

Let us take functions $\gamma(\eta), \mu(\xi)$ so that equalities

$$
\widehat{a}(-\eta, \eta)=-\widehat{b}(-\eta, \eta), \quad \widehat{a_{\xi}}(-\eta, \eta)=\widehat{b_{\eta}}(-\eta, \eta), \quad \eta \leq 0 .
$$

hold. Then from (4.4) we have the following system of equations:

$$
\left\{\begin{array}{lc}
\frac{\gamma^{\prime}(\eta)}{\gamma(\eta)}=-\frac{\mu^{\prime}(-\eta)}{\mu(-\eta)}-a(-\eta, \eta)-b(-\eta, \eta), & \eta \leq 0, \\
\frac{\gamma^{\prime}(\eta)}{\gamma(\eta)}=\frac{\mu^{\prime}(-\eta)}{\mu(-\eta)}-a_{\xi}(-\eta, \eta)+b_{\eta}(-\eta, \eta), & \eta \leq 0 .
\end{array}\right.
$$

This system (4.5) has a solution that can be written as

$$
\begin{aligned}
& \gamma(\eta)=\exp \left[\frac{1}{2} \int_{0}^{\eta}\left(b_{\eta}(-t, t)-a_{\xi}(-t, t)-a(-t, t)-b(-t, t)\right) d t\right] \\
& \mu(\xi)=\exp \left[\frac{1}{2} \int_{0}^{\xi}\left(-b_{\eta}(t,-t)+a_{\xi}(t,-t)-a(t,-t)-b(t,-t)\right) d t\right] .
\end{aligned}
$$


Thus, if $\gamma(\eta), \mu(\xi)$ are selected in this way, condition (4.4) is satisfied at $\eta \leq 0$. For values $\eta>0$, we continue the function $\gamma(\eta)$ in such a way that it is continuously differentiable and the condition $\gamma(\eta)>0$ is satisfied.

To introduce the Riemann-Green function at all points of the domain $\Omega \times \Omega$, we continue the coefficients of equation (4.2) in the domain $\Omega^{-}=\left\{(\xi, \eta) \in \mathbb{R}^{2}: \eta<-|\xi|\right\}$ as follows:

$$
\begin{aligned}
& A(\xi, \eta)= \begin{cases}\widehat{a}(\xi, \eta), & (\xi, \eta) \in \Omega, \\
-\widehat{b}(-\eta,-\xi), & (\xi, \eta) \in \Omega^{-},\end{cases} \\
& B(\xi, \eta)= \begin{cases}\widehat{b}(\xi, \eta), & (\xi, \eta) \in \Omega, \\
-\widehat{a}(-\eta,-\xi), & (\xi, \eta) \in \Omega^{-},\end{cases} \\
& C(\xi, \eta)= \begin{cases}\widehat{c}(\xi, \eta), & (\xi, \eta) \in \Omega, \\
\widehat{c}(-\eta,-\xi), & (\xi, \eta) \in \Omega^{-} .\end{cases}
\end{aligned}
$$

If the coefficients $a(\xi, \eta), b(\xi, \eta) \in C^{1}(\bar{\Omega}) ; c(\xi, \eta) \in C(\bar{\Omega})$, then in virtue of (4.3), (4.4) coefficients $A(\xi, \eta), B(\xi, \eta), C(\xi, \eta)$ in the domain $\widetilde{\Omega}=\Omega \cup \Omega^{-}=\left\{(\xi, \eta) \in \mathbb{R}^{2}: \xi>\eta\right\}$ have the following smoothness:

$$
A(\xi, \eta), B(\xi, \eta) \in C^{1}(\bar{\Omega}) ; \quad C(\xi, \eta) \in C(\bar{\Omega})
$$

and satisfy the following symmetry conditions:

$$
\begin{aligned}
& A(\xi, \eta)=-B(-\eta,-\xi), \quad A_{\xi}(\xi, \eta)=B_{\eta}(-\eta,-\xi), \\
& C(\xi, \eta)=C(-\eta,-\xi), \quad(\xi, \eta) \in \widetilde{\Omega} .
\end{aligned}
$$

Actually, show that (4.10) is true. From (4.6) we have that

$$
\begin{aligned}
A(-\eta,-\xi) & = \begin{cases}\widehat{a}(-\eta,-\xi), & (-\eta,-\xi) \in \Omega, \\
-\widehat{b}(\xi, \eta), & (-\eta,-\xi) \in \Omega^{-},\end{cases} \\
& =-\left\{\begin{array}{ll}
\widehat{b}(\xi, \eta), & (\xi, \eta) \in \Omega, \\
-\widehat{a}(-\eta,-\xi), & (\xi, \eta) \in \Omega^{-},
\end{array}=-B(\xi, \eta) .\right.
\end{aligned}
$$

Also in the same way, from (4.7), (4.8) we get

$$
\begin{aligned}
& A_{\xi}(\xi, \eta)=\left\{\begin{array}{ll}
\widehat{a_{\xi}}(\xi, \eta), & (\xi, \eta) \in \Omega, \\
\widehat{b_{\eta}}(-\eta,-\xi), & (\xi, \eta) \in \Omega^{-},
\end{array}=B_{\eta}(-\eta,-\xi),\right. \\
& C(\xi, \eta)=\left\{\begin{array}{ll}
\widehat{c}(\xi, \eta), & (\xi, \eta) \in \Omega, \\
\widehat{c}(-\eta,-\xi), & (\xi, \eta) \in \Omega^{-},
\end{array}=C(-\eta,-\xi) .\right.
\end{aligned}
$$

If we have chosen $(\xi, \eta)$ from $\Omega$, then $(-\eta,-\xi)$ will be from $\Omega^{-}$. 
In $\widetilde{\Omega}$ we consider the equation

$$
\frac{\partial^{2} U}{\partial \xi \partial \eta}+A(\xi, \eta) \cdot \frac{\partial U}{\partial \xi}+B(\xi, \eta) \cdot \frac{\partial U}{\partial \eta}+C(\xi, \eta) \cdot U=F, \quad(\xi, \eta) \in \widetilde{\Omega}
$$

Due to smoothness (4.8), it is well known that for equation (4.11) the Riemann-Green function [26] exists in $\widetilde{\Omega}$, that for any $(\xi, \eta) \in \widetilde{\Omega}$ satisfies the equation

$$
\begin{aligned}
& \frac{\partial^{2}}{\partial \xi_{1} \partial \eta_{1}} R\left(\xi, \eta ; \xi_{1}, \eta_{1}\right)-\frac{\partial}{\partial \xi_{1}}\left(A\left(\xi_{1}, \eta_{1}\right) R\left(\xi, \eta ; \xi_{1}, \eta_{1}\right)\right) \\
& \quad-\frac{\partial}{\partial \eta_{1}}\left(B\left(\xi_{1}, \eta_{1}\right) R\left(\xi, \eta ; \xi_{1}, \eta_{1}\right)\right)+C\left(\xi_{1}, \eta_{1}\right) R\left(\xi, \eta ; \xi_{1}, \eta_{1}\right)=0, \quad\left(\xi_{1}, \eta_{1}\right) \in \widetilde{\Omega}
\end{aligned}
$$

and the conditions on the characteristics

$$
\begin{aligned}
& \frac{\partial R\left(\xi, \eta ; \xi_{1}, \eta_{1}\right)}{\partial \eta_{1}}-A\left(\xi_{1}, \eta_{1}\right) \cdot R\left(\xi, \eta ; \xi_{1}, \eta_{1}\right)=0, \quad \text { when } \xi_{1}=\xi \\
& \frac{\partial R\left(\xi, \eta ; \xi_{1}, \eta_{1}\right)}{\partial \xi_{1}}-B\left(\xi_{1}, \eta_{1}\right) \cdot R\left(\xi, \eta ; \xi_{1}, \eta_{1}\right)=0, \quad \text { when } \eta_{1}=\eta \\
& R(\xi, \eta ; \xi, \eta)=1 .
\end{aligned}
$$

Thus, with this choice of the method of continuation of the coefficients of equation (4.10), we have determined the values of the Riemann-Green function for all points of the domain $\Omega \times \Omega$.

Lemma 4.1 If conditions (4.10) hold, then the Riemann-Green function has a symmetry such that

$$
R\left(\xi, \eta ; \xi_{1}, \eta_{1}\right)=R\left(-\eta,-\xi ;-\eta_{1},-\xi_{1}\right), \quad(\xi, \eta) \in \widetilde{\Omega},\left(\xi_{1}, \eta_{1}\right) \in \widetilde{\Omega} .
$$

Proof Denote

$$
R_{1}\left(\xi, \eta ; \xi_{1}, \eta_{1}\right)=R\left(-\eta,-\xi ;-\eta_{1},-\xi_{1}\right), \quad(\xi, \eta) \in \widetilde{\Omega},\left(\xi_{1}, \eta_{1}\right) \in \widetilde{\Omega} .
$$

Show that $R_{1}\left(\xi, \eta ; \xi_{1}, \eta_{1}\right)$ satisfies equation (4.12) and conditions (4.13)-(4.15). Indeed, substituting the representation of $R_{1}\left(\xi, \eta ; \xi_{1}, \eta_{1}\right)$ in equation (4.12), at first entering a new notation $-\xi_{1}=\eta_{2},-\eta_{1}=\xi_{2}$, and then also entering the new symbols

$$
-\eta=\widetilde{\xi}, \quad-\xi=\tilde{\eta}, \quad \xi_{2}=\widetilde{\xi}_{1}, \quad \eta_{2}=\tilde{\eta_{1}}
$$


again and using conditions (4.10), we get

$$
\begin{aligned}
\frac{\partial^{2}}{\partial \xi_{1} \partial \eta_{1}} R\left(-\eta,-\xi ;-\eta_{1},-\xi_{1}\right)-\frac{\partial}{\partial \xi_{1}}\left(A\left(\xi_{1}, \eta_{1}\right) R\left(-\eta,-\xi ;-\eta_{1},-\xi_{1}\right)\right) \\
\quad-\frac{\partial}{\partial \eta_{1}}\left(B\left(\xi_{1}, \eta_{1}\right) R\left(-\eta,-\xi ;-\eta_{1},-\xi_{1}\right)\right)+C\left(\xi_{1}, \eta_{1}\right) R\left(-\eta,-\xi ;-\eta_{1},-\xi_{1}\right) \\
=\frac{\partial^{2}}{\partial \xi_{2} \partial \eta_{2}} R\left(-\eta,-\xi ; \xi_{2}, \eta_{2}\right)+\frac{\partial}{\partial \eta_{2}}\left(A\left(-\eta_{2},-\xi_{2}\right) R\left(-\eta,-\xi ; \xi_{2}, \eta_{2}\right)\right) \\
\quad+\frac{\partial}{\partial \xi_{2}}\left(B\left(-\eta_{2},-\xi_{2}\right) R\left(-\eta,-\xi ; \xi_{2}, \eta_{2}\right)\right)+C\left(-\eta_{2},-\xi_{2}\right) \cdot R\left(-\eta,-\xi ; \xi_{2}, \eta_{2}\right) \\
=\frac{\partial^{2}}{\partial \widetilde{\xi_{1}} \partial \widetilde{\eta_{1}}} R\left(\widetilde{\xi}, \widetilde{\eta} ; \widetilde{\xi_{1}}, \widetilde{\eta_{1}}\right)-\frac{\partial}{\partial \widetilde{\eta_{1}}}\left(B\left(\widetilde{\xi_{1}}, \widetilde{\eta_{1}}\right) R\left(\widetilde{\xi}, \widetilde{\eta} ; \widetilde{\xi_{1}}, \widetilde{\eta_{1}}\right)\right) \\
\quad-\frac{\partial}{\partial \widetilde{\xi_{1}}}\left(A\left(\widetilde{\xi_{1}}, \widetilde{\eta_{1}}\right) R\left(\widetilde{\xi}, \widetilde{\eta} ; \widetilde{\xi}_{1}, \widetilde{\eta_{1}}\right)\right)+C\left(\widetilde{\xi_{1}}, \widetilde{\eta_{1}}\right) \cdot R\left(\widetilde{\xi}, \widetilde{\eta} ; \widetilde{\xi_{1}}, \widetilde{\eta_{1}}\right)=0 .
\end{aligned}
$$

Thus $R_{1}\left(\xi, \eta ; \xi_{1}, \eta_{1}\right)$ satisfies equation (4.12). Also substituting the representation of $R_{1}\left(\xi, \eta ; \xi_{1}, \eta_{1}\right)$ into conditions (4.13)-(4.15) and using all the notation at the top, we have

$$
\begin{aligned}
& -\frac{\partial R\left(-\eta,-\xi ;-\eta_{1},-\xi_{1}\right)}{\partial \xi_{1}}-A\left(\xi_{1}, \eta_{1}\right) \cdot R\left(-\eta,-\xi ;-\eta_{1},-\xi_{1}\right) \\
& =\frac{\partial R\left(\widetilde{\xi}, \widetilde{\eta} ; \widetilde{\xi}_{1}, \tilde{\eta}_{1}\right)}{\partial \widetilde{\xi}_{1}}-B\left(\widetilde{\xi_{1}}, \widetilde{\eta}_{1}\right) \cdot R\left(\widetilde{\xi}, \widetilde{\eta} ; \widetilde{\xi_{1}}, \widetilde{\eta_{1}}\right)=0, \quad \text { when } \widetilde{\eta_{1}}=\widetilde{\eta} \text {; } \\
& -\frac{\partial R\left(-\eta,-\xi ;-\eta_{1},-\xi_{1}\right)}{\partial \eta_{1}}-B\left(\xi_{1}, \eta_{1}\right) \cdot R\left(-\eta,-\xi ;-\eta_{1},-\xi_{1}\right) \\
& =\frac{\partial R\left(\widetilde{\xi}, \widetilde{\eta} ; \widetilde{\xi}_{1}, \tilde{\eta}_{1}\right)}{\partial \widetilde{\eta}_{1}}-A\left(\widetilde{\xi}_{1}, \widetilde{\eta}_{1}\right) \cdot R\left(\widetilde{\xi}, \widetilde{\eta} ; \tilde{\xi}_{1}, \widetilde{\eta}_{1}\right)=0, \quad \text { when } \widetilde{\xi_{1}}=\widetilde{\xi} ; \\
& R(-\eta,-\xi ;-\eta,-\xi)=R(\tilde{\xi}, \widetilde{\eta} ; \widetilde{\xi}, \widetilde{\eta})=1 \text {. }
\end{aligned}
$$

Due to (4.17)-(4.20) we see that the function $R\left(-\eta,-\xi ;-\eta_{1},-\xi_{1}\right)$ satisfies the same conditions as the Riemann-Green function of equation (4.11). It is well known that since the Riemann-Green function is a solution to Goursat problem that is correct, then it is unique. It follows that equality (4.16) is true.

Corollary 4.2 On the line $\xi=-\eta, \eta \leq 0$, the following equality holds:

$$
R\left(-\eta, \eta ; \xi_{1}, \eta_{1}\right)=R\left(-\eta, \eta ;-\eta_{1},-\xi_{1}\right) .
$$

\section{Green's function of problem (2.4)-(2.6)}

Let us define a Green's function to the first initial-boundary value problem in the quarter plane:

$$
\begin{aligned}
& \frac{\partial^{2} U}{\partial \xi \partial \eta}+A(\xi, \eta) \cdot \frac{\partial U}{\partial \xi}+B(\xi, \eta) \cdot \frac{\partial U}{\partial \eta}+C(\xi, \eta) \cdot U=F, \quad(\xi, \eta) \in \Omega, \\
& U(\xi, \xi)=T_{1}(\xi), \quad\left(\frac{\partial U}{\partial \xi}-\frac{\partial U}{\partial \eta}\right)(\xi, \xi)=M_{1}(\xi), \quad \xi>0, \\
& U(-\eta, \eta)=P(\eta), \quad \eta \leq 0 .
\end{aligned}
$$


Definition 5.1 The Green's function of problem (5.1)-(5.3) is the function $G\left(\xi, \eta ; \xi_{1}, \eta_{1}\right)$, which for every fixed $\left(\xi_{1}, \eta_{1}\right) \in \Omega$ satisfies the homogeneous equation

$$
L_{(\xi, \eta)} G\left(\xi, \eta ; \xi_{1}, \eta_{1}\right)=0, \quad(\xi, \eta) \in \Omega, \text { at } \xi \neq \xi_{1}, \eta \neq \eta_{1}, \eta \neq-\xi_{1}
$$

and the following boundary conditions:

$$
\begin{aligned}
& G\left(\xi, \xi ; \xi_{1}, \eta_{1}\right)=0, \quad \xi \geq 0,\left(\xi_{1}, \eta_{1}\right) \in \Omega ; \\
& \left(\frac{\partial G}{\partial \xi}-\frac{\partial G}{\partial \eta}\right)\left(\xi, \xi ; \xi_{1}, \eta_{1}\right)=0, \quad \xi \geq 0,\left(\xi_{1}, \eta_{1}\right) \in \Omega, \text { at } \xi \neq \xi_{1}, \eta \neq \eta_{1} ; \\
& G\left(-\eta, \eta ; \xi_{1}, \eta_{1}\right)=0, \quad \eta \leq 0,\left(\xi_{1}, \eta_{1}\right) \in \Omega,
\end{aligned}
$$

and on the above characteristic lines, the following conditions must hold: the values of the derivatives of the Green's function in directions parallel to these characteristics must coincide in adjacent domains; i.e.,

$$
\begin{aligned}
& \frac{\partial G\left(\xi_{1}+0, \eta ; \xi_{1}, \eta_{1}\right)}{\partial \eta}+A\left(\xi_{1}, \eta\right) G\left(\xi_{1}+0, \eta ; \xi_{1}, \eta_{1}\right) \\
& \quad=\frac{\partial G\left(\xi_{1}-0, \eta ; \xi_{1}, \eta_{1}\right)}{\partial \eta}+A\left(\xi_{1}, \eta\right) G\left(\xi_{1}-0, \eta ; \xi_{1}, \eta_{1}\right), \quad \text { at } \eta \neq \eta_{1} ; \\
& \frac{\partial G\left(\xi, \eta_{1}+0 ; \xi_{1}, \eta_{1}\right)}{\partial \xi}+B\left(\xi, \eta_{1}\right) G\left(\xi, \eta_{1}+0 ; \xi_{1}, \eta_{1}\right) \\
& \quad=\frac{\partial G\left(\xi, \eta_{1}-0 ; \xi_{1}, \eta_{1}\right)}{\partial \xi}+B\left(\xi, \eta_{1}\right) G\left(\xi, \eta_{1}-0 ; \xi_{1}, \eta_{1}\right), \quad \text { at } \xi \neq \xi_{1} ; \\
& \frac{\partial G\left(\xi,-\xi_{1}+0 ; \xi_{1}, \eta_{1}\right)}{\partial \xi}+B\left(\xi,-\xi_{1}\right) G\left(\xi,-\xi_{1}+0 ; \xi_{1}, \eta_{1}\right) \\
& \quad=\frac{\partial G\left(\xi,-\xi_{1}-0 ; \xi_{1}, \eta_{1}\right)}{\partial \xi}+B\left(\xi,-\xi_{1}\right) G\left(\xi,-\xi_{1}-0 ; \xi_{1}, \eta_{1}\right) ;
\end{aligned}
$$

and the "corner condition"

$$
\begin{aligned}
& G\left(\xi_{1}-0, \eta_{1}-0 ; \xi_{1}, \eta_{1}\right)-G\left(\xi_{1}+0, \eta_{1}-0 ; \xi_{1}, \eta_{1}\right) \\
& \quad+G\left(\xi_{1}+0, \eta_{1}+0 ; \xi_{1}, \eta_{1}\right)-G\left(\xi_{1}-0, \eta_{1}+0 ; \xi_{1}, \eta_{1}\right)=1
\end{aligned}
$$

must be satisfied as the regions meet at $(\xi, \eta)=\left(\xi_{1}, \eta_{1}\right)$.

\section{Existence and uniqueness of the Green's function of problem (2.4)-(2.6)}

Theorem 6.1 The function $G\left(\xi, \eta ; \xi_{1}, \eta_{1}\right)$ that satisfies conditions (5.4)-(5.11) exists and is unique.

Proof To show that the function $G\left(\xi, \eta ; \xi_{1}, \eta_{1}\right)$ which satisfies conditions (5.4)-(5.11) exists and is unique, we divide the domain $\Omega$ into several subdomains (see Fig. 6) and consider the following problems sequentially. Let $\left(\xi_{1}, \eta_{1}\right)$ be an arbitrary point of the domain $\Omega$. Consider the case of $\eta_{1}>0$, the case of $\eta_{1}<0$ is considered similarly.

In the domain $\Omega_{1}=\left\{(\xi, \eta): 0<\xi<\eta_{1},-\xi<\eta<\xi\right\}$ we consider the problem

$$
L_{(\xi, \eta)} G=0, \quad(\xi, \eta) \in \Omega_{1}
$$




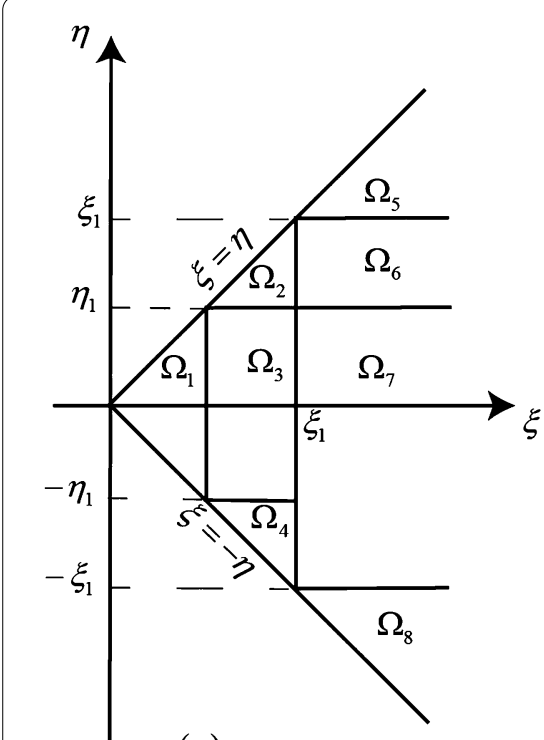

(a)

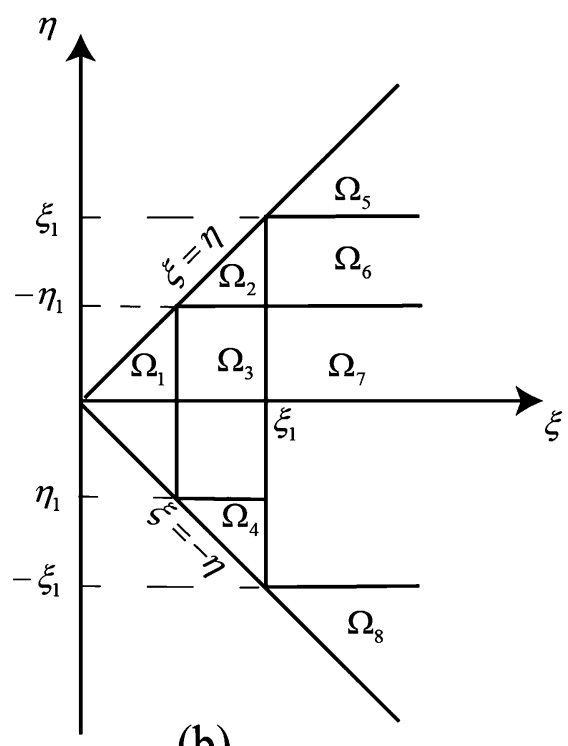

(b)

Figure 6 (a)—splitting the domain $\Omega$, when $\eta_{1}>0$; (b) —splitting the domain $\Omega$, when $\eta_{1}<0$

$$
\begin{aligned}
& G\left(\xi, \xi ; \xi_{1}, \eta_{1}\right)=0, \quad \xi \geq 0 ; \\
& \left(\frac{\partial G}{\partial \xi}-\frac{\partial G}{\partial \eta}\right)\left(\xi, \xi ; \xi_{1}, \eta_{1}\right)=0, \quad \xi \geq 0 ; \\
& G\left(-\eta, \eta ; \xi_{1}, \eta_{1}\right)=0, \quad \eta \leq 0 .
\end{aligned}
$$

Problem (6.1)-(6.4) is the Cauchy-Dirichlet problem and has a unique solution

$$
G\left(\xi, \eta ; \xi_{1}, \eta_{1}\right) \equiv 0, \quad(\xi, \eta) \in \Omega_{1}
$$

In the domain $\Omega_{2}=\left\{(\xi, \eta): \eta_{1}<\xi<\xi_{1}, \eta_{1}<\eta<\xi\right\}$, let us consider the problem

$$
\begin{aligned}
& L_{(\xi, \eta)} G=0, \quad(\xi, \eta) \in \Omega_{2} ; \\
& G\left(\xi, \xi ; \xi_{1}, \eta_{1}\right)=0, \quad \xi \geq 0 ; \\
& \left(\frac{\partial G}{\partial \xi}-\frac{\partial G}{\partial \eta}\right)\left(\xi, \xi ; \xi_{1}, \eta_{1}\right)=0, \quad \xi \geq 0 .
\end{aligned}
$$

Problem (6.6)-(6.8) is the Cauchy problem and has a unique solution

$$
G\left(\xi, \eta ; \xi_{1}, \eta_{1}\right) \equiv 0, \quad(\xi, \eta) \in \Omega_{2}
$$

Therefore from (5.9), (6.5), (6.9) in the domain $\Omega_{3}=\left\{(\xi, \eta): \eta_{1}<\xi<\xi_{1},-\eta_{1}<\eta<\eta_{1}\right\}$, we get the problem

$$
\begin{aligned}
& L_{(\xi, \eta)} G=0, \quad(\xi, \eta) \in \Omega_{3} ; \\
& G\left(\eta_{1}, \eta ; \xi_{1}, \eta_{1}\right)=0, \quad-\eta_{1} \leq \eta \leq \eta_{1}
\end{aligned}
$$




$$
\frac{\partial G\left(\xi, \eta_{1}-0 ; \xi_{1}, \eta_{1}\right)}{\partial \xi}+B\left(\xi, \eta_{1}\right) \cdot G\left(\xi, \eta_{1}-0 ; \xi_{1}, \eta_{1}\right)=0, \quad \eta_{1}<\xi<\xi_{1} .
$$

Integrating (6.12) by $\xi$, we have

$$
G\left(\xi, \eta_{1}-0 ; \xi_{1}, \eta_{1}\right)=\exp \left(-\int_{\eta_{1}}^{\xi} B\left(t, \eta_{1}\right) d t\right) C_{1}\left(\xi_{1}, \eta_{1}\right), \quad \eta_{1}<\xi<\xi_{1} .
$$

Substituting $\xi=\eta_{1}-0$ in (6.13), using condition (5.5), we have that $C_{1}\left(\xi_{1}, \eta_{1}\right) \equiv 0$ and

$$
G\left(\xi, \eta_{1}-0 ; \xi_{1}, \eta_{1}\right)=0, \quad \eta_{1} \leq \xi \leq \xi_{1}
$$

Therefore, problem (6.10)-(6.12) is equivalent to problem (6.10), (6.11), (6.14), which is the Goursat problem and has a unique solution

$$
G\left(\xi, \eta ; \xi_{1}, \eta_{1}\right) \equiv 0, \quad(\xi, \eta) \in \Omega_{3}
$$

Since the Green's function is continuous for $\eta=-\eta_{1}$, then from (6.15) in the domain $\Omega_{4}=\left\{(\xi, \eta): \eta_{1}<\xi<\xi_{1},-\xi<\eta<-\eta_{1}\right\}$ we get the problem

$$
\begin{aligned}
& L_{(\xi, \eta)} G=0, \quad(\xi, \eta) \in \Omega_{4} ; \\
& G\left(-\eta, \eta ; \xi_{1}, \eta_{1}\right)=0, \quad \eta \leq 0 ; \\
& G\left(\xi,-\eta_{1} ; \xi_{1}, \eta_{1}\right)=0, \quad \eta_{1} \leq \xi \leq \xi_{1} .
\end{aligned}
$$

Problem (6.16)-(6.18) is the Darboux problem and has a unique solution

$$
G\left(\xi, \eta ; \xi_{1}, \eta_{1}\right) \equiv 0, \quad(\xi, \eta) \in \Omega_{4} .
$$

In the domain $\Omega_{5}=\left\{(\xi, \eta): \xi_{1}<\xi, \eta>\xi_{1}\right\}$ our problem is the Cauchy problem

$$
\begin{aligned}
& L_{(\xi, \eta)} G=0, \quad(\xi, \eta) \in \Omega_{5} ; \\
& G\left(\xi, \xi ; \xi_{1}, \eta_{1}\right)=0, \quad \xi \geq 0 ; \\
& \left(\frac{\partial G}{\partial \xi}-\frac{\partial G}{\partial \eta}\right)\left(\xi, \xi ; \xi_{1}, \eta_{1}\right)=0, \quad \xi \geq 0,
\end{aligned}
$$

which has a unique solution

$$
G\left(\xi, \eta ; \xi_{1}, \eta_{1}\right) \equiv 0, \quad(\xi, \eta) \in \Omega_{5}
$$

Therefore from (5.8), (6.9), (6.23) in the domain $\Omega_{6}=\left\{(\xi, \eta): \xi_{1}<\xi, \eta_{1}<\eta<\xi_{1}\right\}$ we have the following problem:

$$
\begin{aligned}
& L_{(\xi, \eta)} G=0, \quad(\xi, \eta) \in \Omega_{6} \\
& G\left(\xi, \xi_{1} ; \xi_{1}, \eta_{1}\right)=0, \quad \xi \geq \xi_{1} \\
& \frac{\partial G\left(\xi_{1}+0, \eta ; \xi_{1}, \eta_{1}\right)}{\partial \eta}+A\left(\xi_{1}, \eta\right) G\left(\xi_{1}+0, \eta ; \xi_{1}, \eta_{1}\right)=0, \quad \eta_{1}<\eta<\xi_{1} .
\end{aligned}
$$


Integrating (6.26) by $\eta$, we get

$$
G\left(\xi_{1}+0, \eta ; \xi_{1}, \eta_{1}\right)=\exp \left(-\int_{\eta_{1}}^{\eta} A\left(\xi_{1}, t\right) d t\right) C_{2}\left(\xi_{1}, \eta_{1}\right), \quad \eta_{1}<\eta<\xi_{1} .
$$

Substituting $\eta=\xi_{1}+0$ in (6.27), using condition (5.5), we have that $C_{2}\left(\xi_{1}, \eta_{1}\right) \equiv 0$ and

$$
G\left(\xi_{1}+0, \eta ; \xi_{1}, \eta_{1}\right)=0, \quad \eta_{1} \leq \eta \leq \xi_{1} .
$$

Therefore, problem (6.24)-(6.26) is equivalent to problem (6.24), (6.25), (6.28), which is the Goursat problem and has a unique solution

$$
G\left(\xi, \eta ; \xi_{1}, \eta_{1}\right) \equiv 0, \quad(\xi, \eta) \in \Omega_{6}
$$

From (5.8), (5.9), (5.11), (6.15), (6.19), (6.29) in the domain $\Omega_{7}=\left\{(\xi, \eta): \xi_{1}<\xi,-\xi_{1}<\eta<\right.$ $\eta_{1}$ \} we have the problem

$$
\begin{aligned}
& L_{(\xi, \eta)} G=0, \quad(\xi, \eta) \in \Omega_{7} \\
& \frac{\partial G\left(\xi_{1}+0, \eta ; \xi_{1}, \eta_{1}\right)}{\partial \eta}+A\left(\xi_{1}, \eta\right) G\left(\xi_{1}+0, \eta ; \xi_{1}, \eta_{1}\right)=0, \quad-\xi_{1}<\eta<\eta_{1} . \\
& \frac{\partial G\left(\xi, \eta_{1}-0 ; \xi_{1}, \eta_{1}\right)}{\partial \xi}+B\left(\xi, \eta_{1}\right) G\left(\xi, \eta_{1}-0 ; \xi_{1}, \eta_{1}\right)=0, \quad \xi_{1}<\xi . \\
& G\left(\xi_{1}+0, \eta_{1}-0 ; \xi_{1}, \eta_{1}\right)=-1 .
\end{aligned}
$$

Problem (6.30)-(6.33) is the Goursat problem, and it has a unique solution. Therefore, the function $G\left(\xi, \eta ; \xi_{1}, \eta_{1}\right)$ in the domain $\Omega_{7}$ is uniquely defined. Comparing conditions (6.30)-(6.33) with the following conditions

$$
\begin{aligned}
& L_{(\xi, \eta)} R=0, \quad(\xi, \eta) \in \widetilde{\Omega} ; \\
& \frac{\partial R\left(\xi, \eta ; \xi_{1}, \eta_{1}\right)}{\partial \xi}+B(\xi, \eta) \cdot R\left(\xi, \eta ; \xi_{1}, \eta_{1}\right)=0, \quad \text { when } \eta=\eta_{1} \\
& \frac{\partial R\left(\xi, \eta ; \xi_{1}, \eta_{1}\right)}{\partial \eta}+A(\xi, \eta) \cdot R\left(\xi, \eta ; \xi_{1}, \eta_{1}\right)=0, \quad \text { when } \xi=\xi_{1} \\
& R\left(\xi_{1}, \eta_{1} ; \xi_{1}, \eta_{1}\right)=1
\end{aligned}
$$

which the Riemann-Green function satisfies, it is easy to get the next equality

$$
G\left(\xi, \eta ; \xi_{1}, \eta_{1}\right)=-R\left(\xi, \eta ; \xi_{1}, \eta_{1}\right), \quad(\xi, \eta) \in \Omega_{7}
$$

Therefore from (6.34) in the domain $\Omega_{8}=\left\{(\xi, \eta): \xi_{1}<\xi,-\xi<\eta<-\xi_{1}\right\}$ we get the problem

$$
\begin{aligned}
& L_{(\xi, \eta)} G=0, \quad(\xi, \eta) \in \Omega_{8} ; \\
& G\left(-\eta, \eta ; \xi_{1}, \eta_{1}\right)=0, \quad \eta \leq 0 ;
\end{aligned}
$$




$$
\begin{aligned}
& \frac{\partial G\left(\xi,-\xi_{1}-0 ; \xi_{1}, \eta_{1}\right)}{\partial \xi}+B\left(\xi,-\xi_{1}\right) G\left(\xi,-\xi_{1}-0 ; \xi_{1}, \eta_{1}\right) \\
& =-\frac{\partial R\left(\xi,-\xi_{1} ; \xi_{1}, \eta_{1}\right)}{\partial \xi}-B\left(\xi,-\xi_{1}\right) R\left(\xi,-\xi_{1}-0 ; \xi_{1}, \eta_{1}\right), \quad \xi_{1}<\xi .
\end{aligned}
$$

Problem (6.35)-(6.37) is the Darboux problem and has the unique solution.

Thus, we have shown that, for any $\left(\xi_{1}, \eta_{1}\right) \in \Omega$ and $(\xi, \eta) \in \Omega$, the Green's function that satisfies conditions (5.4)-(5.11) exists and is unique. The theorem is proved.

Corollary 6.2 In the course of proving the existence of the Green's function, we obtained that $G\left(\xi, \eta ; \xi_{1}, \eta_{1}\right) \equiv 0$ in the domains $\Omega_{1}, \Omega_{2}, \Omega_{3}, \Omega_{4}, \Omega_{5}, \Omega_{6}$. That is, $G\left(\xi, \eta ; \xi_{1}, \eta_{1}\right) \equiv 0$ when $\xi_{1}>\xi$.

\section{Construction of the Green's function of problem (2.4)-(2.6)}

As can be seen from the proof of Theorem 6.1, the Green's function $G\left(\xi, \eta ; \xi_{1}, \eta_{1}\right)=0$ in the domains $\Omega_{1}, \Omega_{2}, \Omega_{3}, \Omega_{4}, \Omega_{5}, \Omega_{6}$. And in the domain $\Omega_{7}$ it coincides with the Riemann function (6.34). Let us find a representation of the Green's function in the domain $\Omega_{8}$. To construct the Green's functions, we assume that the coefficients of equation (7.1) satisfy the symmetry conditions of (4.10).

Let $\left(\xi_{1}, \eta_{1}\right)$ be an arbitrary point of the domain $\Omega$. In order to construct the Green's function in the domain $\widetilde{\Omega}_{8}$, consider the problem

$$
\frac{\partial^{2} G_{1}}{\partial \xi \partial \eta}+A(\xi, \eta) \frac{\partial G_{1}}{\partial \xi}+B(\xi, \eta) \frac{\partial G_{1}}{\partial \eta}+C(\xi, \eta) G_{1}=0, \quad(\xi, \eta) \in \widetilde{\Omega}_{8}
$$

where $\widetilde{\Omega}_{8}=\Omega_{8} \cup \Omega_{8}^{-}, \Omega_{8}^{-}=\left\{(\xi, \eta): \xi_{1}<\xi, \eta<-\xi\right\}$,

$$
\begin{aligned}
& \frac{\partial G_{1}\left(\xi,-\xi_{1}-0 ; \xi_{1}, \eta_{1}\right)}{\partial \xi}+B\left(\xi,-\xi_{1}\right) G_{1}\left(\xi,-\xi_{1}-0 ; \xi_{1}, \eta_{1}\right) \\
& \quad=-\frac{\partial R\left(\xi,-\xi_{1} ; \xi_{1}, \eta_{1}\right)}{\partial \xi}-B\left(\xi,-\xi_{1}\right) R\left(\xi,-\xi_{1} ; \xi_{1}, \eta_{1}\right), \quad \xi_{1}<\xi ; \\
& \frac{\partial G_{1}\left(\xi_{1}+0, \eta ; \xi_{1}, \eta_{1}\right)}{\partial \eta}+A\left(\xi_{1}, \eta\right) G_{1}\left(\xi_{1}+0, \eta ; \xi_{1}, \eta_{1}\right) \\
& \quad=\frac{\partial R\left(-\eta,-\xi_{1} ; \xi_{1}, \eta_{1}\right)}{\partial \eta}+A\left(\xi_{1}, \eta\right) R\left(-\eta,-\xi_{1} ; \xi_{1}, \eta_{1}\right), \quad \eta<-\xi_{1} ; \\
& G_{1}\left(\xi_{1},-\xi_{1} ; \xi_{1}, \eta_{1}\right)=0 .
\end{aligned}
$$

Problem (7.1)-(7.4) is the Goursat problem. Its solution exists and is unique. We are interested in the representation of the function $G_{1}\left(\xi, \eta ; \xi_{1}, \eta_{1}\right)$.

Lemma 7.1 If the function $G_{1}\left(\xi, \eta ; \xi_{1}, \eta_{1}\right)$ is a solution to problem (7.1)-(7.4), then for any $(\xi, \eta) \in \widetilde{\Omega}_{8}$ we have $G_{1}\left(\xi, \eta ; \xi_{1}, \eta_{1}\right)=-G_{1}\left(-\eta,-\xi ; \xi_{1}, \eta_{1}\right)$.

Proof To show that the function $-G_{1}\left(-\eta,-\xi ; \xi_{1}, \eta_{1}\right)$ satisfies equation (7.1), in (7.1) replace $\xi=-\eta_{2}, \eta=-\xi_{2},\left(-\eta_{2},-\xi_{2}\right) \in \Omega_{8}^{-}$and after using conditions (4.6)-(4.8), we get that $-G_{1}\left(-\eta,-\xi ; \xi_{1}, \eta_{1}\right)$ satisfies equation (7.1). 
Also making the substitution of $\xi=-\eta_{2}, \eta_{2}<-\xi_{1}$ in (7.2) and using conditions (4.6), (4.7), we get condition (7.3). Similarly, by replacing $-\eta=\xi_{2}, \eta<-\xi_{1}$ in (7.3) and using conditions (4.6), (4.7), we get condition (7.2).

Thus, we have shown that the function $-G_{1}\left(-\eta,-\xi ; \xi_{1}, \eta_{1}\right)$ is also a solution to problem (7.1)-(7.3). Since the solution to problem (7.1)-(7.4) is unique, then

$$
G_{1}\left(\xi, \eta ; \xi_{1}, \eta_{1}\right)=-G_{1}\left(-\eta,-\xi ; \xi_{1}, \eta_{1}\right), \quad(\xi, \eta) \in \widetilde{\Omega}_{8}
$$

We search the solution to problem (7.1)-(7.4) in the following form:

$$
G_{1}\left(\xi, \eta ; \xi_{1}, \eta_{1}\right)=g\left(\xi, \eta ; \xi_{1}, \eta_{1}\right)-R\left(\xi, \eta ; \xi_{1}, \eta_{1}\right), \quad(\xi, \eta) \in \widetilde{\Omega}_{8}
$$

Then, for the function $g\left(\xi, \eta ; \xi_{1}, \eta_{1}\right)$, we get the following problem:

$$
\begin{aligned}
& \frac{\partial^{2} g}{\partial \xi \partial \eta}+A(\xi, \eta) \frac{\partial g}{\partial \xi}+B(\xi, \eta) \frac{\partial g}{\partial \eta}+C(\xi, \eta) g=0, \quad(\xi, \eta) \in \widetilde{\Omega}_{8} ; \\
& \frac{\partial g\left(\xi,-\xi_{1} ; \xi_{1}, \eta_{1}\right)}{\partial \xi}+B\left(\xi,-\xi_{1}\right) g\left(\xi,-\xi_{1} ; \xi_{1}, \eta_{1}\right)=0, \quad \xi_{1}<\xi ; \\
& \frac{\partial g\left(\xi_{1}, \eta ; \xi_{1}, \eta_{1}\right)}{\partial \eta}+A\left(\xi_{1}, \eta\right) g\left(\xi_{1}, \eta ; \xi_{1}, \eta_{1}\right) \\
& \quad=\frac{\partial R\left(-\eta,-\xi_{1} ; \xi_{1}, \eta_{1}\right)}{\partial \eta}+A\left(\xi_{1}, \eta\right) R\left(-\eta,-\xi_{1} ; \xi_{1}, \eta_{1}\right), \quad \eta<-\xi_{1} ; \\
& g\left(\xi_{1},-\xi_{1} ; \xi_{1}, \eta_{1}\right)=R\left(\xi_{1},-\xi_{1} ; \xi_{1}, \eta_{1}\right) .
\end{aligned}
$$

It is easy to see that the solution to problem (7.5)-(7.8) has the form

$$
g\left(\xi, \eta ; \xi_{1}, \eta_{1}\right)=R\left(-\eta,-\xi ; \xi_{1}, \eta_{1}\right), \quad(\xi, \eta) \in \widetilde{\Omega}_{8}
$$

Then from (7.9) we get

$$
G_{1}\left(\xi, \eta ; \xi_{1}, \eta_{1}\right)=R\left(-\eta,-\xi ; \xi_{1}, \eta_{1}\right)-R\left(\xi, \eta ; \xi_{1}, \eta_{1}\right), \quad(\xi, \eta) \in \widetilde{\Omega}_{8} .
$$

Thus the following theorem is proved.

Theorem 7.2 The Green's function of equation (5.1)-(5.3) exists and is unique. This Green's function can be expressed by means of the Riemann-Green function:

$$
\begin{aligned}
& G\left(\xi, \eta ; \xi_{1}, \eta_{1}\right) \equiv 0, \quad \text { if }(\xi, \eta) \in \Omega_{1}, \Omega_{2}, \Omega_{3}, \Omega_{4}, \Omega_{5}, \Omega_{6} \\
& G\left(\xi, \eta ; \xi_{1}, \eta_{1}\right)=-R\left(\xi, \eta ; \xi_{1}, \eta_{1}\right), \quad \text { if }(\xi, \eta) \in \Omega_{7} ; \\
& G\left(\xi, \eta ; \xi_{1}, \eta_{1}\right)=R\left(-\eta,-\xi ; \xi_{1}, \eta_{1}\right)-R\left(\xi, \eta ; \xi_{1}, \eta_{1}\right), \quad \text { if }(\xi, \eta) \in \Omega_{8} .
\end{aligned}
$$

It is well known that for self-adjoint problems (for example, for elliptic equation), the Green's function is symmetric with respect to external and internal variables. In our case, this is not the case for the Green's function of the hyperbolic first initial-boundary value problem. 
Lemma 7.3 Let $(\xi, \eta)$ be an arbitrary point of the domain $\Omega$. By internal variables $\left(\xi_{1}, \eta_{1}\right)$ the Green's function of problem (5.1)-(5.3) has the following properties:

$$
\begin{aligned}
& L_{\left(\xi_{1}, \eta_{1}\right)}^{*} G\left(\xi, \eta ; \xi_{1}, \eta_{1}\right)=0, \quad\left(\xi_{1}, \eta_{1}\right) \in \Omega, \text { at } \xi_{1} \neq \xi, \eta_{1} \neq \eta, \xi_{1} \neq-\eta ; \\
& G\left(\xi, \eta ; \xi_{1},-\xi_{1}\right)=0, \quad \xi_{1}<-\eta ; \\
& \frac{\partial G\left(\xi, \eta ; \xi-0, \eta_{1}\right)}{\partial \eta_{1}}-A\left(\xi, \eta_{1}\right) G\left(\xi, \eta ; \xi-0, \eta_{1}\right)=0, \quad \text { at } \eta_{1} \neq \eta ; \\
& \frac{\partial G\left(\xi, \eta ; \xi_{1}, \eta+0\right)}{\partial \xi_{1}}-B\left(\xi_{1}, \eta\right) G\left(\xi, \eta ; \xi_{1}, \eta+0\right)=0, \quad \text { at } \xi_{1} \neq \xi ; \\
& \frac{\partial G\left(\xi, \eta ;-\eta-0, \eta_{1}\right)}{\partial \xi_{1}}-B\left(-\eta, \eta_{1}\right) G\left(\xi, \eta ;-\eta-0, \eta_{1}\right) \\
& \quad=\frac{\partial G\left(\xi, \eta ;-\eta+0, \eta_{1}\right)}{\partial \xi_{1}}-B\left(-\eta, \eta_{1}\right) G\left(\xi, \eta ;-\eta+0, \eta_{1}\right) \\
& G(\xi, \eta ; \xi-0, \eta-0)-G(\xi, \eta ; \xi+0, \eta-0) \\
& \quad+G(\xi, \eta ; \xi+0, \eta+0)-G(\xi, \eta ; \xi-0, \eta+0)=1 .
\end{aligned}
$$

Proof Properties (7.11)-(7.16) can be easily obtained from the construction of the Green's function of problem (5.1)-(5.3). Under these properties it is possible to uniquely restore the Green's function of problem (5.1)-(5.3).

Using properties (7.11)-(7.16), we can use it to write the integral representation of the solution to problem (5.1)-(5.3). To do this, we consider the following integral:

$$
\begin{aligned}
& \iint_{\Omega_{(\xi \eta)}} G\left(\xi, \eta ; \xi_{1}, \eta_{1}\right) F\left(\xi_{1}, \eta_{1}\right) d \xi_{1} d \eta_{1} \\
& \quad=\iint_{\Omega_{(\xi \eta)}} G\left(\xi, \eta ; \xi_{1}, \eta_{1}\right)\left(\frac{\partial^{2} U}{\partial \xi_{1} \partial \eta_{1}}+A \frac{\partial U}{\partial \xi_{1}}+B \frac{\partial U}{\partial \eta_{1}}+C U\right) d \xi_{1} d \eta_{1} .
\end{aligned}
$$

Applying the Green's theorem in a plane [27] and using initial conditions (5.2), properties of the Green's function, from (7.17) we get the following representation of the solution to problem (5.1)-(5.3) in the domain $\Omega_{(\xi \eta)}$, for $\eta>0$ :

$$
\begin{aligned}
U(\xi, \eta)= & -\frac{1}{2} G(\xi, \eta ; \eta, \eta) T_{1}(\eta)-\frac{1}{2} G(\xi, \eta ; \xi, \xi) T_{1}(\xi) \\
& -\frac{1}{2} \int_{\xi}^{\eta}\left(\frac{\partial G}{\partial N_{1}}\left(\xi, \eta ; \xi_{1}, \xi_{1}\right)+2(A-B)\left(\xi_{1}, \xi_{1}\right) G\left(\xi, \eta ; \xi_{1}, \xi_{1}\right)\right) T_{1}\left(\xi_{1}\right) d \xi_{1} \\
& +\frac{1}{2} \int_{\xi}^{\eta} G\left(\xi, \eta ; \xi_{1}, \xi_{1}\right) M_{1}\left(\xi_{1}\right) d \xi_{1} \\
& +\iint_{\Omega_{(\xi \eta)}} G\left(\xi, \eta ; \xi_{1}, \eta_{1}\right) F\left(\xi_{1}, \eta_{1}\right) d \xi_{1} d \eta_{1} .
\end{aligned}
$$

Also, for $\eta<0$, applying the Green's theorem in a plane [27] and using initial conditions (5.2), boundary condition (5.3), properties of the Green's function, from (7.17) we get the 
following representation of the solution to problem (5.1)-(5.3) in the domain $\Omega_{(\xi \eta)}$ :

$$
\begin{aligned}
U(\xi, \eta)= & \frac{1}{2}(G(\xi, \eta ;-\eta-0, \eta)-2 G(\xi, \eta ;-\eta+0, \eta)) P(\eta) \\
& -\frac{1}{2}(G(\xi, \eta ;-\eta-0,-\eta-0)-G(\xi, \eta ;-\eta+0,-\eta+0)) T_{1}(-\eta) \\
& -\frac{1}{2} G(\xi, \eta ; \xi, \xi) T_{1}(\xi)+\frac{1}{2} \int_{0}^{-\eta} \frac{\partial G}{\partial N_{1}}\left(\xi, \eta ; \xi_{1},-\xi_{1}\right) P\left(-\xi_{1}\right) d \xi_{1} \\
& +\frac{1}{2} \int_{0}^{\xi}\left(\frac{\partial G}{\partial N_{1}}\left(\xi, \eta ; \xi_{1}, \xi_{1}\right)+2(A-B)\left(\xi_{1}, \xi_{1}\right) G\left(\xi, \eta ; \xi_{1}, \xi_{1}\right)\right) T_{1}\left(\xi_{1}\right) d \xi_{1} \\
& -\frac{1}{2} \int_{0}^{\xi} G\left(\xi, \eta ; \xi_{1}, \xi_{1}\right) M_{1}\left(\xi_{1}\right) d \xi_{1}+\iint_{\Omega_{(\xi \eta)}} G\left(\xi, \eta ; \xi_{1}, \eta_{1}\right) F\left(\xi_{1}, \eta_{1}\right) d \xi_{1} .
\end{aligned}
$$

It is easy to see that (7.18), (7.19) are the solutions to problem (5.1), (5.2), (5.3). Substituting $U(\xi, \eta), \gamma(\eta), \mu(\xi)$ for (4.1), we get the solution to problem (2.4)-(2.6).

In conclusion, we would like to note that we have some experience in constructing Green's functions for elliptic problems [28-31]. But, as we learned from this study, the hyperbolic Green's function differs significantly from the Green's functions for elliptic or parabolic problems. In particular, the Green's function of the hyperbolic problem can have discontinuities along several characteristics of the equation. As we can see, in this regard, for each hyperbolic problem, the definition and substantiation of the Green's function must be carried out separately, and detailed studies in this direction are required.

\section{Acknowledgements}

The authors would like to thank T. S. Kalmenov's referee for the invaluable suggestions that improved the presentation of this paper

Funding

The research is financially supported by a grant from the Ministry of Science and Education of the Republic of Kazakhstan (No. AP09561656).

\section{Abbreviations}

Not applicable.

Availability of data and materials

Not applicable.

\section{Competing interests}

The authors declare that they have no competing interests.

\section{Authors' contributions}

This work was carried out in collaboration between both authors. MS designed the study and guided the research. BD performed the analysis and wrote the first draft of the manuscript. MS and BD managed the analysis of the study. Both authors read and approved the final manuscript.

\section{Publisher's Note}

Springer Nature remains neutral with regard to jurisdictional claims in published maps and institutional affiliations.

Received: 1 June 2021 Accepted: 14 July 2021 Published online: 03 August 2021

\section{References}

1. Tyn Myint, U., Lokenath, D.: Linear Partial Differential Equations for Scientists and Engineers. Birkhäuser, Boston (2007)

2. Kreith, K.: Sturmian theorems for hyperbolic equations. Proc. Am. Math. Soc. 22, 277-281 (1969)

3. Kreith, K: A Sturm theorem for partial differential equations of mixed type. Proc. Am. Math. Soc. 81, 75-78 (1981)

4. Kreith, K.: A class of comparison theorems for nonlinear hyperbolic initial value problems. Proc. R. Soc. Edinb. A 87, 190-191 (1981)

5. Swanson, C.A.: A dichotomy of PDE Sturmian theory. SIAM Rev. 20, 285-300 (1978) 
6. Sh, K.T.: On the spectrum of a self-adjoint problem for the wave equation. Vestn. Akad. Nauk Kaz. SSR 2, 63-66 (1982)

7. Sh, K.T.: Spectrum of a boundary - value problem with translation for the wave equation. Differ. Equ. 19(1), 64-66 (1983)

8. Orynbasarov, E.M., Sadybekov, M.A.: The basis property of the system of eigen- and associated functions of a boundary value problem with shift for the wave equation. Math. Notes 51(5-6), 482-484 (1992)

9. Sadybekov, M.A. Orynbasarov, E.M.: Baseness of the system of the eigenfunctions and associated functions with displacement of Lavrentev-Bitsadze equation. Dokl. Akad. Nauk 324(6), 1152-1154 (1992)

10. Yessirkegenov, N.A., Sadybekov, M.A.: Spectral properties of boundary-value problem with a shift for wave equation. Russ. Math. (Izv. VUZ) 60(3), 41-46 (2016)

11. Kreith, K.: A class of hyperbolic focal point problems. Hiroshima Math. J. 14, $203-210$ (1984)

12. Krasnoselskii, M.A.: Positive Solutions of Operator Equations. Noordhoff, Groningen (1964)

13. Kreith, K.: Symmetric Green's functions for a class of CIV boundary value problems. Can. Math. Bull. 31, 272-279 (1988)

14. Kreith, K.: Establishing hyperbolic Green's functions via Leibniz's rule. SIAM Rev. 33, 101-105 (1991)

15. Kreith, K.: A self-adjoint problem for the wave equation in higher dimensions. Comput. Math. Appl. 21, 129-132 (1991)

16. Kreith, K:: Mixed self-adjoint boundary conditions for the wave equation. In: Differential Equations and Its Applications (Budapest). Colloq. Math. Soc. Janos Bolyai, vol. 62, pp. 219-226. North-Holland, Amsterdam (1991)

17. Iraniparast, N.: A method of solving a class of CIV boundary value problems. Can. Math. Bull. 35(3), 371-375 (1992)

18. Iraniparast, N.: A boundary value problem for the wave equation. Int. J. Math. Math. Sci. 22(4), 835-845 (1999)

19. Iraniparast, N.: A CIV boundary value problem for the wave equation. Appl. Anal. 76(3-4), 261-271 (2000)

20. Haws, L:: Symmetric Green's functions for certain hyperbolic problems. Comput. Math. Appl. 21(5), 65-78 (1991)

21. Iraniparast, N.: Boundary value problems for a two-dimensional wave equation. J. Comput. Appl. Math. 55, 349-356 (1994)

22. Iraniparast, N.: A selfadjoint hyperbolic boundary-value problem. Electron. J. Differ. Equ. Conf. 10, 153-161 (2003)

23. Khurshudyan, A.Z.: Nonlinear Green's functions for wave equation with quadratic and hyperbolic potentials. Adv. Math. Phys. 2018, 1-9 (2018)

24. López Molina, J.A.: Green's function for the one-dimensional hyperbolic heat equation: remarks for global regularity. Bol. Soc. Mat. Mexicana 26, 657-671 (2020)

25. Polidoro, S., Ragusa, M.A.: Sobolev-Morrey spaces related to an ultraparabolic equation. Manuscr. Math. 96, 371-392 (1998)

26. Sobolev, S.L.: Equations of Mathematical Physics. Nauka, Moscow (1966)

27. Riley, K.F., Hobson, M.P., Bence, S.J.: Mathematical Methods for Physics and Engineering. Cambridge University Press, Cambridge (2010)

28. Sadybekov, M.A., Turmetov, T.K., Torebek, B.T.: On an explicit form of the Green function of the Robin problem for the Laplace operator in a circle. Adv. Pure Appl. Math. 6(3), 163-172 (2015)

29. Sadybekov, M.A., Torebek, B.T., Turmetov, B.K.: Construction of Green's function of the Neumann problem in a ball. Eurasian Math. J. 7(2), 100-105 (2016)

30. Sadybekov, M.A., Torebek, B.T., Turmetov, B.K.: Representation of Green's function of the Neumann problem for a multi-dimensional ball. Complex Var. Elliptic Equ. 61(1), 104-123 (2016)

31. Sadybekov, M.A., Torebek, B.T., Turmetov, B.K.: Representation of the Green's function of the exterior Neumann problem for the Laplace operator. Sib. Math. J. 58(1), 153-158 (2017)

\section{Submit your manuscript to a SpringerOpen ${ }^{\circ}$ journal and benefit from:}

- Convenient online submission

- Rigorous peer review

- Open access: articles freely available online

- High visibility within the field

- Retaining the copyright to your article

Submit your next manuscript at $\gg$ springeropen.com 